5-30) and mean IQCODE score was 4.2 (SD 0.6; range 3-5). Mean GAI score was 4.97 (SD 5.32; range 0-20) with Cronbach's alpha 0.92. In comparison, a separate population-based cohort of 205 women aged $65+$ years (mean 74.4; SD 5.5; range 65-86) had a mean GAI score of 2.32 (SD 4.03; range 0-19); a separate clinical sample of 46 male and female psychogeriatric patients (mean age 78.8 years; SD 6.7; range 66-94) had a mean GAI score of 5.22 (SD 5.83). As expected, GAI score was correlated with NPI anxiety severity $(\mathrm{r}=0.31 ; \mathrm{p}=0.0037)$, NPI anxiety distress $(\mathrm{r}=$ $0.32 ; \mathrm{p}=0.0031)$ and GDS $(\mathrm{r}=0.62 ; \mathrm{p}=0.000)$. GAI was also correlated with Barthel score $(\mathrm{r}=-0.2837 ; \mathrm{p}=0.0118)$, and leisure activities score $(r=-0.32 ; p=0.0050)$. Conclusions: The mean levels of clinic attendees' self-reported anxiety were much higher than those reported by normal older volunteers but somewhat lower than those reported by psychogeriatric patients. Higher levels of anxiety were reported by patients with higher levels of physical dependency whereas lower levels of anxiety were reported by patients with greater involvement in leisure activities.

\section{P2-240 DEVELOPMENT OF A NEW SCALE MEASURING PSYCHOLOGICAL IMPACT OF GENETIC SUSCEPTIBILITY TESTING FOR ALZHEIMER'S} DISEASE

Robert A. Stern ${ }^{1}$, Winston W. Chung ${ }^{1}$, Clara A. Chen ${ }^{2}$, L. Adrienne Cupples ${ }^{2}$, J. Scott Roberts ${ }^{3}$, Susan C. Hiraki ${ }^{1}$, Anil K. Nair ${ }^{1}$, Robert C. Green ${ }^{1},{ }^{1}$ Boston University School of Medicine, Boston, MA, USA; ${ }^{2}$ Boston University School of Public Health, Boston, MA, USA; ${ }^{3}$ University of Michigan School of Public Health, Ann Arbor, MI, USA. Contact e-mail: bobstern@bu.edu

Background: With the growing number of individuals with $\mathrm{AD}$ and the even greater number of older Americans at risk for $\mathrm{AD}$, it is likely that genetic susceptibility testing will become an important clinical, ethical, and research issue in the near future. The REVEAL Study (Risk Evaluation and Education for Alzheimer's Disease) is a series of randomized clinical trials designed to evaluate the impact of risk assessment, including APOE genotype disclosure, for AD. The purpose of the present study was to develop a brief, self-report measure of the psychological impact of genetic susceptibility tests for $\mathrm{AD}$ to be used in both clinical (e.g. genetic counseling) settings as well as in research on genetic risk disclosure. Methods: The new instrument, The REVEAL Impact of Genetic Testing for Alzheimer's disease (IGT-AD) was designed to examine the unique nature of genetic information and the disease course of $\mathrm{AD}$. The scale was tested as a part of a multi-center clinical trial designed to evaluate the impact of AD risk assessment and data was collected from 276 participants in the study. Results: Using an iterative process of Principal Component Analysis and Cronbach's alpha, the final 16 item IGT-AD was found to have a two factor structure with excellent internal reliability $(0.82)$. Construct validity was established by patterns of correlation with other standardized self-reported measures, such as the Impact of Event Scale, the Beck Depression Inventory, the Beck Anxiety Inventory, and the Center for Epidemiologic Studies Depression Scale. Conclusions: The IGT-AD scale should be useful in the identification of patients who maybe prone to the negative effects of receiving genetic information, monitoring of patients who have received genetic information, and as a tool for researchers who wish to study the effects of genetic susceptibility testing for AD.

\section{P2-241 ASSESSMENT OF AWARENESS OF COGNITIVE CHANGE IN INDIVIDUALS WITH AND WITHOUT DEMENTIA: THE CACHE COUNTY STUDY}

Trevor Buckley ${ }^{1}$, Maria Norton ${ }^{1}$, Michael Deberard ${ }^{1}$, Kathy Welsh-Bohmer ${ }^{2}$, Constantine Lyketsos ${ }^{3}$, JoAnn Tschanz ${ }^{1},{ }^{1}$ Utah State University, Logan, UT, USA; ${ }^{2}$ Duke University, Durham, NC, USA; ${ }^{3}$ Johns Hopkins University, Baltimore, MD, USA. Contact e-mail: t.buckley@aggiemail.usu.edu

Background: Anosognosia or lack of awareness of impairment may be a prominent feature of dementia. Yet there are few standardized methods of assessment amenable for use in epidemiological research. In the Cache County, UT population, we administered a brief, 7-item measure of selfperception of cognitive change (metacognition). To examine its utility for examining anosognosia in dementia, we compared responses on this measure with two external criteria: longitudinal performance on a cognitive screening test and informant ratings of cognitive and functional decline. We predicted that metacognitive ratings would exhibit lower associations with external criteria in individuals with dementia as opposed to those without dementia. Methods: A total of 687 individuals (152 with incident dementia and 535 without dementia) completed a brief metacognition questionnaire about change in their cognitive and functional abilities over the past 3 years. $99 \%$ of the participants were Caucasian, $57 \%$ were female. Mean (SD) age and education were 82 (7.1) and 13 (3.0) years. All had been administered the Modified Mini-Mental State Exam (3MS) twice, over an approximate 3-year interval, and 490 had an informant ratings of their cognitive and functional ability on the Informant Questionnaire of Cognitive Decline in the Elderly (IQCODE). Correspondence between self-perception and 3MS change was examined with multiple regression; correspondence between self-perception and informant ratings of change or no change was examined with logistic regression. In both models we controlled for demographics and overall health status. Results: Mean metacognition scores indicated little to no perceived change in cognition in $65 \%$ of dementia subjects and $78 \%$ in those without dementia. Metacognition ratings significantly predicted $3 \mathrm{MS}$ change $(\mathrm{p}=0.027)$ and informant ratings $(p=0.029)$ in those without dementia. In those with dementia, there was a marginal, inverse association with $3 \mathrm{MS}$ change $(\mathrm{p}=0.056)$, suggesting poor self-awareness. Informant ratings were not associated with metacognition in this group. Conclusions: We demonstrated the utility of a brief metacognition questionnaire in examining anosognosia in the elderly. Future studies will examine the utility of this questionnaire in predicting conversion of individuals with Mild Cognitive Impairment to dementia.

\section{P2-242 A STANDARDIZATIONAL STUDY FOR THE KOREAN VERSION OF COHEN-MANFIELD AGITATION INVENTORY}

Jeong-Lan Kim, Chungnam National University Hospital, Daejeon, Republic of Korea. Contact e-mail: kimjl@cnu.ac.kr

Background: to standardize and develop the Korean version of CohenMansfield Agitation Inventory (K-CMAI). Methods: After developing the K-CMAI, 166 patients, who met diagnostic criteria of dementia, were rated by it and Korean version of Neuropsychiatric Inventory (K-NPI). Results: Factor analysis from Varimax Rotation of K-CMAI was found 4 factors, such as aggressive behaviors, physically non-aggressive behaviors, verbally agitated behaviors, and hiding and hoarding, and we could confirm those 4 factors with Amos. Irritability, Disinhibition, and aberrant behavior domains of K-NPI were highly correlated with K-CMAI. Conclusions: The K-CMAI was showed good reliability and validity for the assessment of agitated behavior in elderly

\section{P2-243 DEMENTIA SYMPTOMS OF ALZHEIMER'S DISEASE AND VASCULAR DEMENTIA REPORTED BY CAREGIVERS: A PRELIMINARY STUDY FOR THE CONSTRUCTION OF THE KOREAN DEMENTIA QUESTIONNAIRE}

Yeonwook Kang ${ }^{1}$, Jaeseol Park ${ }^{2}$, Kyunghee Ryu ${ }^{3}$, Duk L. Na ${ }^{4},{ }^{1}$ Hallym University, Chuncheon, Gangwondo, Republic of Korea; ${ }^{2}$ Hallym University Sacred Heart Hospital, Anyang, Geonggido, Republic of Korea; ${ }^{3}$ Yonsei University, Seoul, Republic of Korea; ${ }^{4}$ Samsung Medical Center, Seoul, Republic of Korea. Contact e-mail: ykang@hallym.ac.kr

Background: As the number of elderly people increases, so does the number of dementia patients. There has been a stronger imperative than ever to develop the instruments that screen the dementia. The present study was conducted as a preliminary study to construct the Korean Dementia Questionnaire (KDQ) used for screening the dementia in clinical settings or 